\title{
Detection of 10 kind of pesticides multi-residue in fruits and vegetables by LC-MS/MS
}

\author{
Weiming Tang ${ }^{1}$,Shuxiang Sun ${ }^{2}$, Minghong Jia ${ }^{1}$, a , Huijun Liu ${ }^{1}$, Tiwen $\mathrm{Li}^{1}$, \\ Xiaohong Yang ${ }^{1}$,Mengqi Zhang ${ }^{1}$ \\ ${ }^{1}$ Beijing University of Agriculture, Beijing 102206, China; \\ ${ }^{2}$ Henan chemical vocational college, Henan 450042, China; \\ amh_jia@yahoo.com
}

Key words: QuEChERS; LC-MS/MS; fruits and vegetables; pesticide multi-residue

\begin{abstract}
This paper established a multi-residue detection method of amides, neonicotinoids, triazoles, heterocyclic, and other pesticides in fruits and vegetables samples by liquid chromatography - tandem mass spectrometry. Using QuEChERS as sample pretreatment method, then extracted by acetonitrile, cleaned up by graphitized carbon black using dispersed solid phase extraction. After the separation of chromatographic column, the extraction was analyzed under the LC-MS/ MS multiple reaction monitoring mode. Ten kind of pesticides detection is limited between $0.006 \sim 0.133 \mu \mathrm{g} / \mathrm{kg}$, and the quantitative is limited between $0.018 \sim 0.415 \mu \mathrm{g} / \mathrm{kg}$. On the three adding levels between $1 \sim 10 \mu \mathrm{g} / \mathrm{kg}$, the average recovery is $76.2 \% \sim 96.3 \%$, RSD is less than $2.4 \%$. This method can be used in the pesticides multi-residue detection of fruits and vegetables samples.

This article selected amides ${ }^{[1-2]}$, nicotine, carbamate, triazole and methoxyl acrylic ester and other 5 kind of pesticides, boscalid, fluopicolide, flonicamid, desflurane bacteria amide, dinotefuran, epoxiconazole, imidacloprid, thiacloprid, azoxystrobin, isopropyl Granville, and boscalid fungicide belong to amides, mainly applied for controlling gray mold, sclerotinia rot and various other fungi of fruits and vegetables ${ }^{[3-7]}$. Neonicotinoid pesticides dinotefuran are for aphids, planthoppers and other pests ${ }^{[8-9]}$. Carbamate pesticides are widely applied in agriculture, forestry, and animal husbandry ${ }^{[12]}$. Isopropyl Granville are for planthoppers, rice leafhopper, aphids, bugs, etc. ${ }^{[11-12]}$ Azoxystrobin is one strobilurin fungicide first listing, with broad-spectrum, long persistence, and high selection ${ }^{[13-14]}$. The pesticides are featured about high efficiency and broad spectrum, which has been widely applied in the fruits and vegetables.

Studies have shown that these pesticides cause some harm to human health, so our country developed its maximum levels for fruits and vegetables in the standard (MRLs), the imidacloprid is $1 \mathrm{mg} / \mathrm{kg}$, organism bacteria acyl amine is $5 \mathrm{mg} / \mathrm{kg}$, fluorine pyrazole bacterium amine is $0.5 \mathrm{mg} / \mathrm{kg}$, desflurane bacteria amide is $0.9 \mathrm{mg} / \mathrm{kg}$, fluorine organism worm amide is $1 \mathrm{mg} / \mathrm{kg}$, fluorine ring azole to $3 \mathrm{mg} / \mathrm{kg}$, its ester is $5 \mathrm{mg} / \mathrm{kg}$, thiamethoxam moiety to $1 \mathrm{mg} / \mathrm{kg}$, isopropyl is $0.5 \mathrm{mg} / \mathrm{kg}$, cefuroxime worm amine is $0.8 \mathrm{mg} / \mathrm{kg}^{\text {[15-16] }}$.

Liquid tandem mass spectrometry, featuring of high sensitivity, high accuracy and high selectivity, has been widely used in multi-component analysis and detection of pesticide multi-residues and become an important means of measuring small molecule compounds in complex matrices ${ }^{[20-21]}$. This article selected apples, grapes, tomatoes, cucumbers, using QuCHERs technology for pretreatment, multiple reaction monitoring mode (MRM) of liquid chromatography-tandem mass spectrometry (LC/MS/MS) for multi-residues detection.

By studying test conditions, the linear range, detection limit, and precision of these five classes of 10 kind of pesticides, this article established a multi-residues detection method by liquid chromatography tandem mass spectrometry, to provide faster, more accurate and more practical method for further study.
\end{abstract}




\section{Experiment}

\subsection{Instruments and reagents}

Liquid chromatography-mass spectrometry(Agilent 1200-ESI 6410, the United States)

H-1850R centrifuge (Changsha Xiang Yi centrifuge machines Ltd.)

AWL-1002-U Echo Pu Ultrapure water system (Chongqing Shun Yang Enterprise Development Co., Ltd.)

BF-2000 nitrogen blow drying apparatus eight square technology co., LTD. (Beijing)

$50 \mathrm{ml}$ plastic centrifuge tube (BD Biosciences company)

HPLC grade acetonitrile (Burdick\&Jackson, Honeywell)

Analysis of pure formic acid, chemical reagent company (Beijing)

Bacteria acyl amine (97.0\% purity), fluorine pyrazole bacteria amine (95.0\%), desflurane bacteria amide standard (98.0\%), were purchased from Shenzhen Rowe agrochemical co., LTD. Thiamethoxam moiety $(99.5 \%$, CHEM SERVICE), fluorine organism worm amide $(96.0 \%$, shaanxi road on grid biological science co., LTD.), its bacteria ester $(99.0 \%)$, imidacloprid $(98.0 \%)$, were purchased from Hebei ChengYue chemical co., LTD. cefuroxime worm amine $(98.0 \%$, shandong joint), fluorine ring azole (97.0\%), isopropy(98.0\%) were purchased from Beijing and shun technology co., LTD.

\subsection{The preparation of the standard solution}

\subsubsection{Standard stock solution}

Respectively put $0.0101 \mathrm{~g}$ bacteria acyl amine and $0.0103 \mathrm{~g}$ desflurane bacteria amide pesticide standard into $100 \mathrm{ml}$ volumetric flask and dissolved by acetone and constant volume; Respectively put $0.0103 \mathrm{~g}$ furbenicillin, $0.0100 \mathrm{~g}$ isopropyl amine , $0.0102 \mathrm{~g}$ fluorine organism worm amide pesticide standard into $100 \mathrm{ml}$ volumetric flask and dissolved by methanol and constant volume; Respectively put $0.0102 \mathrm{~g}$ fluorine pyrazole bacteria amine, $0.0102 \mathrm{~g}$ fluorine ring azole pesticide standard into $100 \mathrm{ml}$ volumetric flask, dissolved by methylene chloride and constant volume; Respectively put $0.0104 \mathrm{~g}$ its bacteria ester, $0.0102 \mathrm{~g}$ thiamethoxam moiety pesticide imidacloprid, $0.0103 \mathrm{~g}$ standard into $100 \mathrm{ml}$ volumetric flask, dissolved by acetonitrile and constant volume, and stored at $-18{ }^{\circ} \mathrm{C}$.

\subsubsection{Mixed standard solution}

Respectively add $5 \mathrm{~mL}$ single pesticide standard stock solution into $100 \mathrm{~mL}$ volumetric flask, then use acetonitrile solution constant volume, configurate $5 \mathrm{ug} / \mathrm{mL}$ mixed standard solution, $4{ }^{\circ} \mathrm{C}$ freezing away from light.

\subsection{Sample treatment}

Weigh $10.00 \mathrm{~g}$ samples into $50 \mathrm{~mL}$ stoppered centrifuge tube, $20 \mathrm{~mL}$ acetonitrile and $5 \mathrm{~g}$ sodium chloride were added, after high-speed homogenization $1 \mathrm{~min}$ and centrifuge $5 \mathrm{~min} 7000 \mathrm{r} / \mathrm{min}$, take $1.5 \mathrm{~mL}$ supernatant into $2 \mathrm{~mL}$ centrifuge tube, $50 \mathrm{mg}$ PSA and $2 \mathrm{~g}$ anhydrous magnesium sulfate was added, shock centrifugal and take $1 \mathrm{~mL}$ supernatant, filtrated through $0.22 \mathrm{um}$ microporous membrane, dried by nitrogen blow, use $1 \mathrm{~mL}$ acetonitrile solution to constant volume and stored..

\subsection{Conditions of chromatography and mass spectrometry}

Conditions of Chromatographic column : Pursuit C18 $(250 \mathrm{~mm} \times 4.6 \mathrm{~mm}, 5 \mathrm{um}$, Agilent companies in the United States); Mobile phase: A is $0.1 \%$ formic acid aqueous solution, B is acetonitrile; Flow rate: $0.4 \mathrm{~mL} / \mathrm{min}$ : column temperature: $30{ }^{\circ} \mathrm{C}$. Injection volume is $5 \mathrm{uL}$.

Conditions of mass spectrometry: electrospray ionization sourcepositive ion mode $(\mathrm{ESI}+)$, the reactive ion detection mode (MRM), the MRM parameter table shown in table 1 . The dry nitrogen gas temperature is $300{ }^{\circ} \mathrm{C}$, velocity of $6 \mathrm{~L} / \mathrm{min}$; Spray atomization gas pressure 137931 pa (20 psi) capillary voltage $4000 \mathrm{v}$. 
Table 1 LC-MS/MS parameters of 10 pesticide

\begin{tabular}{ccccccc}
\hline type & Parent ion & $\begin{array}{c}\text { Quantitative } \\
\text { ion }\end{array}$ & $\begin{array}{c}\text { Qualitative } \\
\text { ion }\end{array}$ & $\begin{array}{c}\mathrm{CE} \\
(\mathrm{eV})\end{array}$ & $\begin{array}{c}\mathrm{FV} \\
(\mathrm{V})\end{array}$ & $\begin{array}{c}\text { Peak time } \\
(\mathrm{min})\end{array}$ \\
\hline $\begin{array}{c}\text { Boscalid } \\
\text { Fluopicolide }\end{array}$ & 343.2 & 307.0 & 139.9 & 15 & 130 & 20.464 \\
Flonicamid & 230.1 & 172.7 & 364.6 & 15 & 130 & 21.291 \\
Fluoride ether & 415.4 & 206.8 & 398.7 & 15 & 130 & 8.596 \\
Bacteria amide & 253.1 & 125.8 & 185.7 & 15 & 90 & 25.531 \\
Thiacloprid & 202.9 & 129.0 & 113.1 & 10 & 135 & 9.744 \\
Dinotefuran & 330.1 & 120.9 & 140.9 & 15 & 135 & 7.739 \\
Epoxiconazole & 194.1 & 95.1 & 137.1 & 10 & 105 & 14.376 \\
Isoprocorb & 256.1 & 175.1 & 209.1 & 15 & 90 & 9.017 \\
Imidacloprid & & & & & & \\
\hline
\end{tabular}

\section{Results and discussion}

\subsection{Optimization of extraction conditions}

Acetonitrile is commonly used in the laboratory extraction agent, easy to volatile and water soluble, after adding the amount of sodium chloride can be separated with water, fat, protein, sugar compounds are not easy to extract, high recovery rate of pesticides. In the analysis of pesticide residue, in order to improve the recovery rate of the easily degradable pesticide in the basic medium, the samples were extracted with acetonitrile.

PSA contains primary amine and secondary amine groups, with weak anion exchange capacity, commonly used in the extraction of liquid dispersed solid phase extraction purification. In the experiment, PSA was used to purify the acetonitrile extract, and PSA was used to preserve the 10 target compounds, so the extraction solution was not needed to be concentrated and purified.

\subsection{Optimization of conditions for liquid chromatography tandem mass spectrometry}

\subsubsection{Selection of mobile phase}

To the best separation effect were investigated. Ammonium acetate - methanol, ammonium acetate acetonitrile and formic acid methanol and formic acid in acetonitrile solution as mobile phase separation. The results showed that in acetonitrile under the condition of income spectrum baseline fluctuations are smaller than those of the fluctuation of methanol and imidacloprid, Fluopicolide and boscalid failed to completely separated under the conditions of methanol, extend the detection time, so the choice of separation effect better acetonitrile as the organic phase. In the water adding formic acid or ammonium acetate is conducive to the ionization of target compounds, due to ammonium acetate than formic acid ionization effect is poor, so formate as inorganic phase, and investigated the effects of the concentration of formic acid solution on the separation effect. It was found that when the acid was $0.1 \%$, the 10 pesticides were completely separated. To sum up, in the $0.1 \%$ formic acid acetonitrile separation effect is the best, so the choice of $0.1 \%$ formic acid acetonitrile as mobile phase. Under optimized conditions, the total ion flow diagram of the 10 pesticides in the standard solution is shown in figure 1.

\subsubsection{Effect of flow rate on separation time}

Experiments were presented to investigate the flow rate on the separation time of impact, within the pressure range of the column, When the flow rate is $/ \mathrm{min} 0.3 \mathrm{~mL}$, the separation and detection of the 10 pesticides need to be $40 \mathrm{~min}$, When the flow rate is transferred to $/ \mathrm{min} 0.4 \mathrm{~mL}$, the flow time of the target compound is ahead of time, and the complete separation can be achieved in the $30 \mathrm{~min}$. 


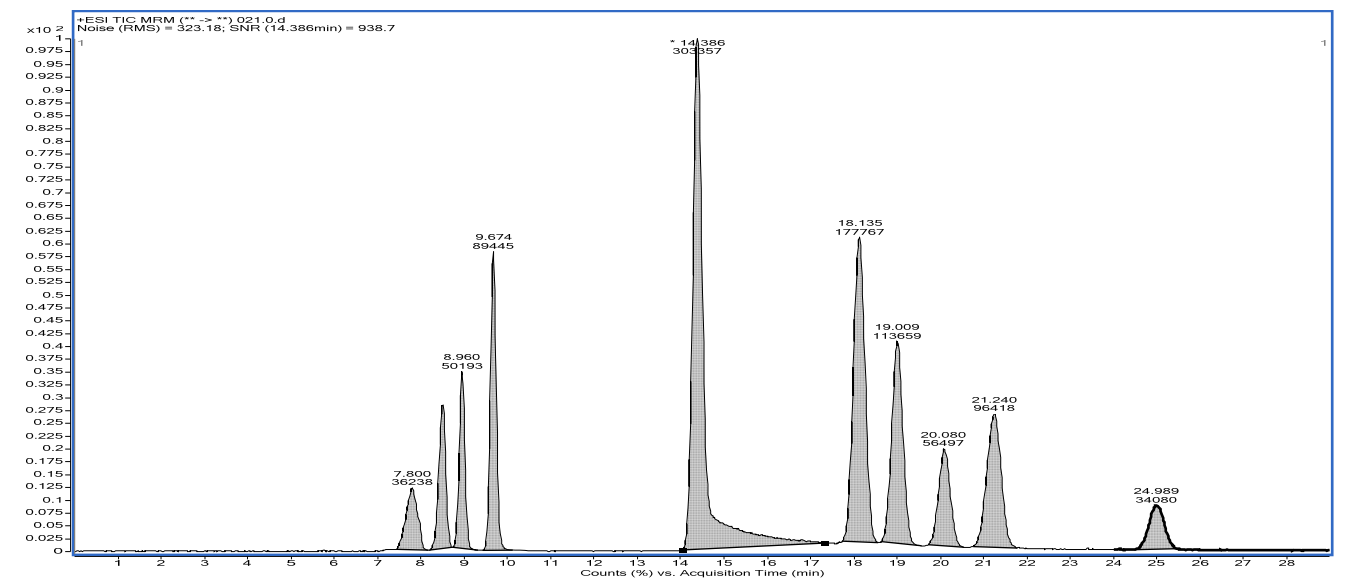

Picture 1 MRM chromatogram under optimized condition

\section{3 linear range and detection limit}

Take a certain amount of mixed standard solution and acetonitrile diluted into standard solution $1,2,5,10,20,50,100 \mathrm{~g} / \mathrm{L}$, in HPLC-MS / MS analysis, the peak area (y) on the quality of compound concentration $(\mathrm{x})$ linear regression, draw the standard curve, 10 kinds of pesticide standard curve linear correlation coefficient (R2) between the 0.9981 0.9997, show that in $1 \sim 100 \mathrm{~g} / 1$ range with good linearity.

The detection limit of the method with the lowest dilution standard curve of blank sample matrix on the peak when $\mathrm{SNR}=3 \mathrm{R} / \mathrm{N}$ calculated. Quantitation limit is in blank sample matrix dilution standard curve, the lowest concentration peak, to win the trust of noise than $\mathrm{R} / \mathrm{N}$ for 10 calculated, pesticide detection and quantitation limits were 0.15 to $0.2 \mathrm{~g} / \mathrm{kg}$ and $0.5 \sim 0.6 \mathrm{u} \mathrm{g} / \mathrm{kg}$.

Table 2 Linear equations and correlation coefficients of 10 pesticides in the sample solution

\begin{tabular}{ccc}
\hline Type & Linear equation & $\mathrm{r}^{2}$ \\
\hline Boscalid & $\mathrm{y}=48920 \mathrm{x}-180.25$ & $\mathrm{r}^{2}=0.9992$ \\
fluopicolide & $\mathrm{y}=88075 \mathrm{x}+1261.8$ & $\mathrm{r}^{2}=0.9989$ \\
flonicamid & $\mathrm{y}=29584 \mathrm{x}-202.34$ & $\mathrm{r}^{2}=0.9992$ \\
Fluoride ether bacteria amide & $\mathrm{y}=28019 \mathrm{x}+85.438$ & $\mathrm{r}^{2}=0.9997$ \\
Thiacloprid & $\mathrm{y}=85809 \mathrm{x}+90.41$ & $\mathrm{r}^{2}=0.9998$ \\
dinotefuran & $\mathrm{y}=17534 \mathrm{x}+62.718$ & $\mathrm{r}^{2}=0.9989$ \\
epoxiconazole & $\mathrm{y}=103805 \mathrm{x}+696.47$ & $\mathrm{r}^{2}=0.9995$ \\
Isoprocorb & $\mathrm{y}=182397 \mathrm{x}+2523.3$ & $\mathrm{r}^{2}=0.9990$ \\
Imidacloprid & $\mathrm{y}=25145 \mathrm{x}-6.1854$ & $\mathrm{r}^{2}=0.9997$ \\
Azoxystrobin & $\mathrm{y}=177501 \mathrm{x}+1705.3$ & $\mathrm{r}^{2}=0.9981$
\end{tabular}


Table 310 kinds of pesticides in apple, grape, tomato, cucumber in the added recovery rate, LOD, LOQ

\begin{tabular}{|c|c|c|c|c|c|c|c|c|c|c|c|}
\hline \multirow{2}{*}{ Type } & \multirow{2}{*}{$\begin{array}{c}\text { Adding } \\
\text { concentratio } \\
\mathrm{n}(\mathrm{mg} * \mathrm{~kg}-1)\end{array}$} & \multicolumn{2}{|c|}{ Apple } & \multicolumn{2}{|c|}{ Grape } & \multicolumn{2}{|c|}{ Tomatoes } & \multicolumn{2}{|c|}{ Cucumber } & \multirow{2}{*}{$\begin{array}{l}\text { LOD } \\
\left(\mu \mathrm{g}^{*} \mathrm{k}\right. \\
\left.\mathrm{g}^{-1}\right)\end{array}$} & \multirow{2}{*}{$\begin{array}{l}\text { LOQ } \\
\left(\mu g^{*} k\right. \\
\left.g^{-1}\right)\end{array}$} \\
\hline & & $\begin{array}{c}\text { rate of } \\
\text { recovery } \\
\%\end{array}$ & $\begin{array}{l}\text { RSD } \\
\%\end{array}$ & $\begin{array}{c}\text { rate of } \\
\text { recovery } \\
\%\end{array}$ & $\begin{array}{l}\text { RSD } \\
\%\end{array}$ & $\begin{array}{c}\text { rate of } \\
\text { recovery } \\
\%\end{array}$ & $\begin{array}{l}\text { RSD } \\
\%\end{array}$ & $\begin{array}{c}\text { rate of } \\
\text { recovery } \\
\%\end{array}$ & $\begin{array}{l}\mathrm{RS} \\
\mathrm{D} \\
\% \\
\end{array}$ & & \\
\hline \multirow[t]{3}{*}{ Boscalid } & 0.01 & 77.6 & 2.3 & 89.6 & 1.6 & 83.6 & 2.9 & 82.1 & 3.1 & \multirow{3}{*}{0.15} & \multirow{3}{*}{0.5} \\
\hline & 0.05 & 80.3 & 1.9 & 88.1 & 1.9 & 81.6 & 1.6 & 84.3 & 1.2 & & \\
\hline & 0.5 & 76.2 & 2.8 & 88.9 & 2.3 & 83.9 & 1.4 & 81.1 & 1.5 & & \\
\hline \multirow[t]{4}{*}{ fluopicolide } & 0.01 & 79.3 & 1.7 & 83.6 & 0.9 & 78.7 & 3.9 & 79.8 & 1.8 & & \\
\hline & 0.05 & 78.8 & 3.3 & 86.4 & 1.5 & 80.3 & 2.5 & 83.4 & 2.8 & 0.15 & 0.5 \\
\hline & 0.5 & 85.1 & 2.1 & 88.2 & 1.2 & 83.4 & 1.3 & 83.3 & 1.4 & & \\
\hline & 0.01 & 89.4 & 2.8 & 90.6 & 1.8 & 87.6 & 4.3 & 89.5 & 1.6 & & \\
\hline \multirow[t]{3}{*}{ flonicamid } & 0.05 & 87.1 & 2.1 & 89.3 & 4.4 & 84.5 & 2.1 & 88.6 & 7.2 & 0.2 & 0.6 \\
\hline & 0.5 & 86.4 & 3.9 & 92.7 & 2.3 & 88.9 & 1.7 & 93.1 & 1.8 & & \\
\hline & 0.01 & 85.6 & 1.3 & 83.1 & 0.8 & 79.2 & 1.4 & 81.4 & 0.9 & & \\
\hline \multirow{3}{*}{$\begin{array}{l}\text { Fluoride ether } \\
\text { bacteria amide }\end{array}$} & 0.05 & 86.9 & 0.8 & 85.6 & 1.6 & 78.4 & 2.4 & 82.8 & 2.1 & 0.15 & 0.5 \\
\hline & 0.5 & 85.3 & 1.5 & 88.1 & 3.6 & 80.3 & 1.2 & 80.7 & 3.2 & & \\
\hline & 0.01 & 80.6 & 1.6 & 79.6 & 1.8 & 80.9 & 3.5 & 82.6 & 1.6 & & \\
\hline \multirow[t]{3}{*}{ Thiacloprid } & 0.05 & 86.1 & 2.5 & 82.3 & 1.5 & 81.8 & 1.1 & 84.1 & 1.2 & 0.2 & 0.6 \\
\hline & 0.5 & 88.3 & 1.4 & 81.2 & 2.9 & 77.6 & 4.1 & 80.8 & 4.6 & & \\
\hline & 0.01 & 87.3 & 4.1 & 87.7 & 1.3 & 86.8 & 0.9 & 90.8 & 1.4 & & \\
\hline \multirow[t]{3}{*}{ dinotefuran } & 0.05 & 89.6 & 1.6 & 90.3 & 1.6 & 88.4 & 2.1 & 89.6 & 1.9 & 0.2 & 0.6 \\
\hline & 0.5 & 92.1 & 1.2 & 91.2 & 5.2 & 85.2 & 1.3 & 89.9 & 1.1 & & \\
\hline & 0.01 & 79.8 & 2.2 & 87.2 & 1.9 & 80.1 & 3.7 & 80.2 & 4.1 & & \\
\hline \multirow[t]{3}{*}{ epoxiconazole } & 0.05 & 82.4 & 1.4 & 85.1 & 1.6 & 78.6 & 1.3 & 79.8 & 2.4 & 0.2 & 0.6 \\
\hline & 0.5 & 83.3 & 1.9 & 88.4 & 3.5 & 79.4 & 6.3 & 78.1 & 1.9 & & \\
\hline & 0.01 & 80.3 & 0.8 & 82.5 & 1.3 & 80.6 & 1.4 & 82.5 & 2.6 & & \\
\hline \multirow[t]{3}{*}{ Isoprocorb } & 0.05 & 82.6 & 1.6 & 85.4 & 2.1 & 82.4 & 2.1 & 84.4 & 0.7 & 0.15 & 0.5 \\
\hline & 0.5 & 83.5 & 6.4 & 86.6 & 1.8 & 79.1 & 1.4 & 81.2 & 1.1 & & \\
\hline & 0.01 & 79.6 & 2.3 & 79.6 & 6.4 & 83.4 & 1.9 & 86.1 & 1.3 & & \\
\hline \multirow[t]{3}{*}{ Imidacloprid } & 0.05 & 87.5 & 1.2 & 83.5 & 1.3 & 79.6 & 1.2 & 89.9 & 3.3 & 0.15 & 0.5 \\
\hline & 0.5 & 83.6 & 2.2 & 84.8 & 2.1 & 81.5 & 4.2 & 85.3 & 1.5 & & \\
\hline & 0.01 & 86.5 & 3.1 & 96.3 & 0.8 & 85.4 & 1.2 & 89.7 & 1.9 & & \\
\hline \multirow[t]{2}{*}{ Azoxystrobin } & 0.05 & 88.9 & 1.6 & 92.1 & 2.8 & 86.9 & 1.9 & 86.5 & 2.2 & 0.15 & 0.5 \\
\hline & 0.5 & 89.7 & 2.1 & 94.4 & 1.3 & 82.2 & 2.3 & 91.2 & 1.8 & & \\
\hline
\end{tabular}

\section{4 recovery and precision of the method}

This method uses tomatoes, grapes, apples and cucumbers 4 kinds of fruit and vegetable as samples, respectively with the addition of $0.001,0.05$ and $0.1 \mathrm{mg} / \mathrm{kg}$ three concentration levels of mixed standard solution, according to the pretreatment method, rate of recovery experiments, each repeated 3 times measured precision. The results are shown in the table.

As we can seen from table, 10 kinds of pesticides in the three levels, The average recovery rate was between $76.2 \% \sim 96.3 \%$, the relative standard deviation (RSD) between $0.8 \% \sim 2.4 \%$, the accuracy and precision of the method are in line with the requirements of residue analysis. According to this method to detect the local supermarket fruits and vegetables samples, including 
apples, grapes, tomatoes and cucumbers in the sample did not exceed the maximum pesticide residue limits standard.

\section{Summary}

In this article,the rapid detection methods of 10 pesticides in 4 fruits and vegetables were successfully established by QuEChERS pretreatment, liquid chromatography separation and tandem quadrupole mass spectrometry.The results show that the method has a simple sample processing, high sensitivity,good reproducibility,qualitative and quantitative results are reliable for apple,grapes,simultaneous determination of 10 kinds of pesticide residues in tomatoes and cucumbers,but also for other fruits and vegetables pesticides in simultaneous determination of residues of reference.

\section{Acknowledgments}

I had a lot of guidance and help thank teacher Jia Minghong, experiment and the met a lot of problems. Every time he would patience guidance, explain, even at the expense of their rest time, the subject was able to proceed smoothly. Not only that, Zhao teacher with their own actions infected us, his rigorous academic attitude, let me see the professor should have the quality to be a. Here to express my heartfelt thanks to jia! Also thanks to the lab teacher Liu Huijun, she in scientific research has a very rich experience, to the subject of my research provides great help, also put forward a lot of suggestions for improvement, so that I benefited a lot.

Also want to thank my brother Li Tiwen, sister Yang Xiaohong liuna and, in the experimental process they help me modify the experiment method, and points out the problems.

Thank you for the test conditions and a good test environment provided by the chemical laboratory of Beijing University of Agriculture, which is the basis of this study.

Finally, thanks to all the teachers and students who have given me help, I will repay you by my own efforts!

Thank you for the experimental conditions and good testing environment, and the "2014 vertical - and - within - suction pesticide testing technology optimization and application in fruits and vegetables (20140131)" project support for the chemical laboratory of Beijing University of Agriculture.

Finally, thanks to all the teachers and students who have given me help, I will repay you by my own efforts!

\section{Reference}

[1] Yi Bing. Novel fungicide Boscalid[J]. World pesticide. 2006， 28(5): 51-53.

[2] Yu Lexiang, Zhang Xiuzhen. Research progress of novel nicotinamide fungicide boscalid[J]. Pesticide information of Shandong, 2009, 12(11): 29-30.

[3] Chen Li, Jia Chunhong, He Min. High performance liquid chromatographic analysis of residues in strawberry and soil[J]. Pesticides, 2009, 48(5): 361-364.

[4] Zhang Yuting, Guo Yongze, Liu Lei. Residue dynamics of the amines in grape and soil[J]. 2011, 50(1): 51-52.

[5] Wang Yunfeng, Wang Wei, Qin Hongjian. Determination of 10 kinds of amide fungicides residues in fruits and vegetables by liquid chromatography - mass spectrometry[J].Food research and development, 2010, 31(1): 113-116.

[6] ANASTASIOS E, HELEN B, SPYROS A, et al. Determination of multi-class pesticides in wines by solid-phase extraction and liquid chromatography-tandem mass spectrometry[J]. J Chromatogr A, 2009, 1216(31): 5856-5867. 
[7] LIU Xinggang, DONG Fengshou, QIN Dongmei, et al. Residue analy- sis of kresoxim-methyl and boscalid in fruits, vegetables and soil using liquid-liquid extraction and gas chromatography-mass spectrometry[J]. Biomedical Chromatography, 2010, 24(4): 367-373.

[8] Li Li, Fu Jiang, Chu Yusheng. Study on HPLC-MS/MS method for detecting the dinotefuran residue in food[J], Science of food , 2008,28(11:538-540)2008, 29 (11) :538 -540.

[9]Xie Wen, Qian Yan, Ding Huiying. Determination of 6 kinds of pesticide residues in tea by liquid chromatography electrospray ionization mass spectrometry and mass spectrometry with four levels and three levels [ J ] . Analytical chemistry, 2009,37

[10]Liang Jiamei. Review and Prospect on the achievements of pesticide application in China Plant protection technology and extension, 2000, 20(3):32-33

[11] Zhu Baoping, Ye Yazhen, Leng Jianrong, Lin Zhuanhong. Application of detection of GC-MS in poison, organophosphorus and carbamate pesticide poison, Practical preventive medicine, 2008, 15( 5) : 1579-1581

[12] Zhu Shuping, Ding Yuejiang, Wang Jianxiu, Xu Guoqiang. Modern preventive medicine with carbamate pesticide aldicarb caused by food poisoning, 2008, 35( 14) : 2658-2664

[13] Li Fugen, Song Junhua, Wang Yiyan. Recent progress in the registration and management of microbial pesticides in the European Union[J]. Pesticides, 2008, 47(9): 629-630.

[14] Yan Xiaojing, Jin Shuhui, Wang Daoquan. Advances in the research of the target of Strobilurin fungicides $[\mathrm{J}]$. Chinese journal of pesticide science, 2006, 8( 4):299 -305.

[15] ZHuang Wuji. Regulations on pesticide residues in food and feed (Volume 1)[M]. Beijing: Chemical Industry Press, 2010: 148-149.

[16] ZHuang Wuj. Regulations on pesticide residues in food and feed in the world (volumes 2)[M]. Bei Jing: Chemical Industry Press2010: 108.

[17]Sun Zhen, Gu Yaping,Qian He.Development of pesticide residue analysis and detection technology in fruits and vegetables[J].Food science and technology,2007,(1):15-19.

[18]Gong Jiuping,Li Yan,Hong Yunju.Optimization of method for determination of amino acid ester pesticide residues in vegetables by high performance liquid chromatography[J ].Southwest China journal of agricultural science,2009,22(5) : 1332-1335.

[19]Chen Ying,Cong Peihua,Nie Jiyun.Study on the detection methods of pesticide residues in fruits and the effect of matrix[J].Analytical laboratory,2008,27 (5):408-412.

[20]Cao Zhaoyun,Mou Renxiang,Ying Yinghua.Determination of 80 pesticide residues in vegetables and fruits by liquid chromatography tandem mass spectrometry[J].Fen xi ce shi xue bao,2010,29(10):1030-1035.

[21]YangHuiqin,Guo Dehua,Li Bo.Determination of 75 pesticide residues in plant products by gel chromatography tandem mass spectrometry[J].Health inspection of China,2007,17(8):1355-1358. 